\title{
"Legs et Littérature", Revue de littérature contemporaine, La critique littéraire, dir. C. Shread
}

\section{Elena Fermi}

\section{(2) OpenEdition}

1 Journals

Édition électronique

URL : https://journals.openedition.org/studifrancesi/21809

DOI : 10.4000/studifrancesi.21809

ISSN : 2427-5856

Éditeur

Rosenberg \& Sellier

\section{Édition imprimée}

Date de publication : 1 décembre 2019

Pagination : 618-619

ISSN : 0039-2944

\section{Référence électronique}

Elena Fermi, « "Legs et Littérature", Revue de littérature contemporaine, La critique littéraire, dir. C. Shread », Studi Francesi [En ligne], 189 (LXIII | III) | 2019, mis en ligne le 01 mars 2020, consulté le 11 novembre 2021. URL : http://journals.openedition.org/studifrancesi/21809 ; DOI : https://doi.org/ 10.4000/studifrancesi.21809

Ce document a été généré automatiquement le 11 novembre 2021.

\section{(c) (†)}

Studi Francesi è distribuita con Licenza Creative Commons Attribuzione - Non commerciale - Non opere derivate 4.0 Internazionale. 


\title{
"Legs et Littérature", Revue de littérature contemporaine, $L a$ critique littéraire, dir. C. Shread
}

\author{
Elena Fermi
}

\section{RÉFÉRENCE}

"Legs et Littérature", Revue de littérature contemporaine, La critique littéraire, dir. C.

Shread, D. Petit Frère, 10, juillet 2017, Haïti, Legs édition, 2017.

1 Ce numéro 10 de la revue Legs et Littérature - publication semestrielle de littérature contemporaine dirigée par Charles Wébert, consacrée en particulier à la littérature des îles - est dédié à la critique littéraire. Il se compose de six sections. La première - qui en constitue le noyau - présente neuf contributions autour du sujet annoncé dans le titre et fera l'objet, par la suite, d'une analyse plus approfondie. La deuxième est constituée de trois témoignages et entretiens. Dans le premier, Darline Alexis et Nadève Ménard débattent au sujet de la critique littéraire en Haïti, en se plaignant de son déficit de cadrage théorique, du manque d'institutions formelles et de l'incompréhension, de la part du public, du rôle qu'elle est appelée à jouer. Stéphane Martelly présente, ensuite, son nouveau livre Les jeux du dissemblable. Folie, marge et féminin en littérature haïtienne contemporaine, paru en 2016 aux éditions Nota Bene. Yanick Lahens s'entretient enfin sur ce qui l'a amenée vers la création littéraire après ses débuts plutôt liés à l'essai critique. La troisième section est constituée de comptes rendus de lecture, tandis que la quatrième nous présente trois ouvrages de création: Le voyageur, un récit en prose de Robert Silivi, Deuil, un poème de Kukouvi Dzifa Galley et Sablier et boussole, encore un poème de James Stanley Jean-Simon. Dans la cinquième partie on peut trouver la relation de Carl-Henry Pierre sur le festival de théâtre En lisant de Port-au Prince, dont la première édition - consacrée à Jean-Marie Koltès - a eu lieu en 2016, ainsi qu'une 
liste de prix, distinctions et événements. La sixième partie est enfin un Repère bibliographique d'œuvres critiques de la littérature haïtienne.

Dans son éditorial introductif, Dieulermesson Petit Frère annonce les axes du discours qui va être présenté dans les neuf articles constituant le cœur du numéro. Les questions par lesquelles il entame sa réflexion donnent la dimension de la difficulté de cerner le sujet: à quoi ressemble la littérature d'Haïti aujourd'hui? Est-il possible désormais d'associer aux nombreuses œuvres de fictions qui en font partie une réflexion plus générale sur la création? Qu'est-ce que la critique littéraire et à quoi sert-elle? En partant des considérations de Jean-Paul Sartre et Tzvetan Todorov - qui définissaient l'objet d'étude de cette discipline comme un phénomène au double statut, social et culturel - il en conclut que la critique tend à cerner le rapport entre le secret de la création et le mystère de la réception (p. 7). Le point de vue haïtien sur la question doit prendre en compte le fait que, dans l'île, l'œuvre littéraire a fait l'objet d'interprétation et d'analyse dès ses premiers moments comme en témoignent les nombreuses publications - revues et journaux - qui sont nées au fil des années pour se consacrer à ce sujet. Dieulermesson évoque ensuite l'œuvre de Thibaudet qui - dans ses Réflexions sur la critique - stipule que «la vraie et complète critique ne naît qu'au XIX ${ }^{e}$ siècle» afin de tracer les limites à l'intérieur desquelles vont s'inscrire les contributions au présent numéro. Il ne s'agit pas - dit-il - de «faire l'inventaire des théories critiques ou des figures de la critique haïtienne» mais de «produire un discours sur la critique littéraire et proposer de nouvelles pistes pour mieux réfléchir sur l'œuvre littéraire» (p. 10). Tout cela, à la lumière de diverses théories et à partir d'un corpus d'œuvres de genres différents.

Loudiyi Mourad se penche sur le roman French Dream de l'écrivain marocain Mohamed Hmoudane. Publié en 2005, il s'agit d'un ouvrage que la critique n'arrive pas à classer dans un genre spécifique et qui fluctue entre autobiographie, confession intime, quête incessante d'une identité qui ne peut être définie que dans un rapport constant à l'altérité, mais aussi entre fiction et imagination. En faisant appel aux concepts de biculturalisme et de transgénéricité, l'auteur analyse l'œuvre de Hmoudane à travers le filtre de la question identitaire. Il en conclut que le lecteur est ici face à un roman où «le discours fictionnel réorganise les faits autobiographiques présentant devant le lecteur un exemple d'un roman transgénérique et la représentation d'un «Je» éclaté, doté d'une identité instable et à reconstruire, cherchant son image dans l'altérité» (p. 36). Alix Émera se tourne vers la figure à la fois fascinante et gênante de Jean-Jacques Dessalines - fondateur de l'État haïtien - afin d'analyser sa représentation et son appropriation par les créateurs et dans les croyances populaires. Son article a comme point de départ des poèmes de circonstance des premiers littérateurs qui font l'éloge de ce héros épique dans la période qui suit la proclamation de l'Indépendance, pour se poursuivre avec la présentation de la lecture que firent de sa figure les historiens de la génération de 1836. Après avoir évoqué le drame La Fille de l'empereur, écrit en 1860 par Liautaud Ethéart, l'auteur termine son propos avec les essayistes et les romanciers qui, $\mathrm{au} \mathrm{Xx}^{\mathrm{e}}$ siècle, ne font désormais dans leurs fictions que quelques brèves allusions à Dessalines en laissant, en revanche, une plus grande place à Toussaint Louverture. Pour ce qui est de la tradition populaire, enfin, Émera affirme ne pas avoir assez d'éléments pour examiner les occurrences du personnage dans ce type de productions même s'il est plausible que des échos de l'importance qu'il a eue dans l'histoire de l'île se trouvent dans certains proverbes, chansons populaires et formules locales. Carrol F. Coates 
consacre sa contribution à l'œuvre romanesque de Jacques Stephen Alexis. Il distingue d'un côté Compère Général Soleil et Les Arbres musiciens où l'auteur crée un contraste entre un gouvernement corrompu et un peuple misérable et, de l'autre, L'Espace d'un cillement et L'Étoile Absinthe où il semble vouloir prévoir une grande Fédération Caraïbe opposée aux États-Unis. Ce qui intéresse le romancier, dans toutes les œuvres du corpus, est - selon le chercheur - de remettre en question la politique nordaméricaine telle qu'elle a été conduite dans son pays. Le but de l'analyse est notamment celui d'amener le lecteur à réfléchir sur la stratégie de masquage de cette politique, mise en place par Alexis derrière les péripéties de ses romans. Eddy Arnold Jean consacre son article à l'œuvre foisonnante de Jean-Claude Fignolé dont il souligne la grandeur, la variété et la profondeur. En faisant le tour de la production romanesque de l'auteur, il montre à quel point, chez lui, la réflexion sur le discours a précédé l'expérimentation de l'écriture et dans quelle mesure le roman devient pour lui la totalité d'une pluralité de discours. C'est encore à un roman, Le choc de Léon Laleau, que Mirline Pierre dédie son article. À travers l'analyse de la manière dont est construit le récit, la chercheuse tente d'en faire ressortir les traits indigénistes afin de montrer comment l'auteur cherche à mettre en question l'occupation américaine de son île et à en appeler à la révolte. Artiste pluridimensionnel, infatigable inventeur de mondes, expert en dynamique syntaxique et pyromane lexical, Frankétienne fait l'objet de l'article de Marie-Josée Desvignes. Elle se penche en particulier sur La marquise sort à cinq heures, œuvre poétique au titre évocateur qui met en scène le périple à la fois érotique et mystique d'une voyageuse solitaire et libre. Selon la chercheuse, le renvoi à Paul Valéry - contenu dans le titre - «met d'emblée le lecteur en position d'adhérer ou pas à cette folie scripturaire, ce mouvement labyrinthique et en spirale» (p. 106) dans lequel le je du poète et celui de la marquise se confondent. Jean James Estépha se tourne vers les anthologies de la littérature haïtienne, afin de s'interroger sur leurs différentes orientations, sur les critères de leurs choix d'auteurs et de textes et sur le réel apport qu'elles donnent à la littérature de l'île. Il en conclut que - toute anthologie étant gouvernée par un souci de sélection - on ne peut pas prétendre une neutralité et une objectivité qui contrastent avec la nature même de ce genre de texte. Suzanne Eyenga Onana conduit une analyse de L'Otage de Faustin Mvogo sous le prisme de l'ethnocritique, afin de mettre en relief l'adéquation profonde entre le signifiant des noms propres des personnages du roman et leur signifié romanesque qui permettrait au romancier, selon l'auteure, d'aborder la problématique politique de son pays, le Cameroun, «par le biais d'une onomastique fertile» (p. 152) et de nommer ainsi les maux dont souffre son pays. L'article de Dieulermesson Petit Frère qui clôt la section se tourne vers l'œuvre de Yanick Lahens, en analysant en particulier les romans La Couleur de l'aube et Guillaume et Nathalie. C'est surtout la célébration de l'idéal féminin que l'auteur entend ici prendre en compte. Selon lui Lahens, en mettant en avant la figure féminine et en lui conférant une grande charge érotique, s'affranchirait de certains tabous sociaux. Son exploration romanesque des bas-fonds de la société haïtienne lui permettrait à la fois de fustiger le snobisme de la classe moyenne, l'avarice de la bourgeoisie mais aussi de nous raconter deux histoires qui concilient les contraires et nous donnent l'espoir d'un monde plus juste. Chacun des articles est suivi d'une bibliographie.

4 La revue, tout en révélant par moments un défaut de supervision, présente toutefois une richesse de contributions qui permet au lecteur de faire un tour assez exhaustif de la question prise en compte. 